\title{
Gene Expression Modulation of Two Biosynthesis Pathways via Signal Transduction in Cochliobolus heterostrophus
}

\author{
Ofir Degani ${ }^{1,2}$ \\ ${ }^{1}$ Tel-Hai College, Tel-Hai, Israel \\ ${ }^{2}$ Migal-Galilee Research Institute, Kiryat Shmona, Israel \\ Email: d-ofir@bezeqint.net, ofird@telhai.ac.il
}

Received 1 December 2013; revised 8 February 2014; accepted 4 March 2014

Copyright (C) 2014 by author and Scientific Research Publishing Inc.

This work is licensed under the Creative Commons Attribution International License (CC BY). http://creativecommons.org/licenses/by/4.0/

(c) (i) Open Access

\section{Abstract}

G-protein-linked pathways have evolved to allow responses to extracellular agonists (hormones, neurotransmitters, odors, chemoattractants, light and nutrients) in eukaryotic cells, ranging from simpler systems, including yeasts, filamentous fungi and slime molds, to more complex organisms, such as mammals. Although the role of G-protein and mitogen-activated protein kinase (MAPK) in filamentous fungi has been studied for over a decade, downstream elements are less known, and the study of target genes has evolved mainly in recent years. Here, we examined the involvement of G-protein subunits and MAPK in controlling the expression of two distinct target genes. These genes were selected from an array database according to their unique expression profile and the role of closely related genes found in other Ascomycetes. One of these genes is $B P H$, which encodes the enzyme responsible for cytochrome P450-dependent benzoate hydroxylation in microsomes. The other gene is CIPA, which encodes isoflavone reductase (IfR), an enzyme involved in the synthesis of phytoalexin, which catalyzes an intermediate step in pisatin biosynthesis. The expression profile of these two genes was determined in a series of signaling deficiency mutants that were grown on different media using a DNA microarray. Comparison of the expression profile in the two wild type strains and mutants deficient in the G-protein $\alpha$ or $\beta$ subunits or in MAPK, revealed a unique control mechanism for the $B P H$ and $C I P A$ genes. The two genes are highly expressed during the infection of the host plant leaves and may associate with the fungal response to the host. Signaling via G-protein or MAPK was shown to be related to cascades that altered the expression of these genes in response to the growth condition. This work demonstrates that signal transduction pathways are controlling genes that, although sharing an environmental dependent response, participate in distinct biosynthesis pathways. Moreover, the transcriptional profile may point to distinct and shared roles of the signaling components. 


\section{Keywords}

\section{Cochliobolus heterostrophus; Cytochrome P450-Dependent Benzoate Hydroxylase; G-Protein; Isoflavone Reductase; Maize; MAPK; Signal Transduction}

\section{Introduction}

The small GTP-binding protein Ras and heterotrimeric G proteins in eukaryotic cells are involved in the transmission of external signals. Filamentous fungi recognize and respond to signals from the environment and host organisms by altering their growth and development. In Cochliobolus heterostrophus, the agent of Southern Corn Leaf Blight, G $\alpha 1$ (CGA1) and $\mathrm{G} \beta$ (CGB1) subunits, as well as of a mitogen-activated protein kinase (MAPK, CHK1), participates in several developmental pathways. Disruption studies of these signaling components results in severe phenotypes, including loss of the normal meandering hyphal growth pattern on hard surfaces, lack of appressorium formation, and defects in mating and virulence [1]-[6]. Both GTP-G $\alpha$ and free G $\beta \gamma$ can activate downstream targets [7], and an epistatic relationship may exist between the two branches of the G-protein pathway [5].

A MAPK module is a known target of G $\beta \gamma$ in budding yeast and mammalian cells [8] [9], and new evidence indicates that some crosstalk may exist between the MAPK and the G-protein pathways in C. heterostrophus as well [5]. Data on G-protein subunits and MAPK involvement in the regulation of gene expression in fungal species are constantly growing. Nevertheless, only a few genes have been shown to be regulated by signal transduction in C. heterostrophus to date. Among these are two cellulase genes whose expression is modulated by the MAPK pathway [10] [11] and four hydrophobins [12]. In addition, the expression of key genes involved in melanin biosynthesis in two C. heterostrophus MAPKs, CHK1 and MPS1, has been shown to be induced significantly under hyperosmotic conditions compared to invariably high expression in the wild type (WT) [13].

Here, we examined the expression variations resulting from the disruption of G-protein $\alpha$ and $\beta$ subunits and of MAPK in two distinct genes. The two genes were selected from a microarray database according to their unique expression profile and the role of closely related genes found in other Ascomycetes. The first gene, designated CIPA, showed high homology in the deduced amino acid sequence (Blastx: Expect $=1 \mathrm{e}-54$, in $549 \mathrm{bp}$ ) to the 2'-hydroxyisoflavone reductase (IfR; EC 1.3.1.45) family protein CipA from Aspergillus fumigatus/nidulans and to other fungi hypothetical proteins (Gibberella zeae, Neurospora crassa and Magnaporthe grisea). Phytoalexins are low molecular weight antimicrobial compounds synthesized by plants in response to attempted infection by fungal pathogens, exposure to elicitor macromolecules, or other biotic and abiotic stresses [14]-[17]. Isoflavonoid phytoalexins are characteristic of Leguminosae and are very rarely found in other plant families [18]. The pterocarpan medicarpin is the major phytoalexin in the alfalfa forage legume. It is biosynthesized in two pathways, one of them (the isoflavonoid branch pathway) involved specific enzymes that include IfR. This enzyme catalyzes the NADPH-dependent reduction of 2'-hydroxyformononetin to vestitone, which is the penultimate step in the synthesis of medicarpin [19]. IfR protein has been purified and characterized from several legumes, and IFR cDNA clones have been obtained from alfalfa [20], chickpea [21] and pea [16]. In unstressed alfalfa plants, transcripts from the single alfalfa IFR gene are detected mainly in roots and root nodules, consistent with the accumulation of a medicarpin conjugate, medicarpin-3-O-glucoside-6'-Omalonate, only in these organs [20]. IFR transcripts are, however, strongly induced in infected leaves or elicited cell cultures at the onset of medicarpin accumulation [22]. In fungi, CipA is a member of the protein family associated with the response to the antibiotic concanamycin A produced by Streptomyces sp. [23]. In Candida sp., a CIP1 gene with little homology with IFR of plants, was proposed to play a crucial role in the establishment of a specific cellular response to stress resulting from the cadmium treatment [24]. These observations may indicate that the specific role of IFR in the synthesis of defense-related isoflavonoid compounds may be recruited to defense needs also in fungi.

The second gene chosen for this study, $B P H$, showed high homology in the amino acid sequence (BLASTX: Expect $=9 \mathrm{e}-177$, in $1605 \mathrm{bp}$ ) to cytochrome P450 benzoate 4-monooxygenase (Benzoate-para-hydroxylase, $\mathrm{BpH}$, EC 1.14.13.12.) from A. fumigatus and other fungi (N. crassa, Phanerochaete chrysosporium and Rhodotorula minuta), and to other hypothetical fungi proteins (G. zeae, Ustilago maydis and M. grisea). Cytochromes 
P450 (CYP) are a super family of enzymes crucial for the oxidative, peroxidative and reductive metabolism of a diverse group of compounds, including endobiotics, such as steroids, bile acids, fatty acids, prostaglandins and leukotrienes, and xenobiotics, including most of the therapeutic drugs and environmental pollutants [25]. CYP enzymes are expressed ubiquitously in different life forms: they have been found in animals, plants, fungi and bacteria [25]. They seem to be indispensable for eukaryotic species, but not for prokaryotes, since some bacteria lack CYP enzymes [27]. Eukaryotics need CYPs for the biosynthesis of sterols, which are constituents of plasma membrane [26]. Eukaryotic CYP enzymes are membrane-bound, mostly localized to the endoplasmic reticulum, but some CYPs are also present in mitochondrial inner membranes. In order to function, cytochrome P450s require an electron transfer chain. In the endoplasmic reticulum, this source is NADPH-cytochrome P450 reductase, previously called NADPH-cytochrome $\mathrm{C}$ reductase [26]. In mitochondria, electrons are transferred from NADPH by redoxin reductase to redoxin and then to CYP [28]. Despite their occasionally minimal sequence similarity, all CYPs have a similar structural fold with a highly conserved core [29].

P450rm has been well conserved during the evolution of fungi as a benzoate 4-hydroxylase in the dissimilation pathway starting from L-phenylalanine [30]. The enzyme catalyzed the reaction: Benzoate $+\mathrm{NADPH}+\mathrm{H}^{+}$ $+\mathrm{O}_{2}=$ 4-hydroxybenzoate $+\mathrm{NADP}^{+}+\mathrm{H}_{2} \mathrm{O}$ and requires $\mathrm{P} 450$ reductase (CprA) as the mediator in electron donation from NADPH. In the endoplasmic reticulum of the filamentous fungus A. niger, a cytochrome P450 enzyme system is present that is capable of the para-hydroxylation of benzoate [31]. The A. niger gene called CYP53 was defined to be a member of the cytochrome P450 sub family, P450LIII (CYP53 family). The expression of the two genes encoding the components of this system, the cytochrome P450 gene encoding benzoate para-hydroxylase A (BpHA) and the gene encoding cytochrome P450 CprA, is inducible by benzoate [31]. The $B P H A$ orthologous gene from A. nidulans, BZUA, was localized in the endoplasmic reticulum and was identified as being required for the utilization of benzamide as the sole nitrogen source [32]. The BZUA1 mutation prevents the use of benzoate as a carbon source, and intracellular accumulation of benzoate results in growth inhibition on benzamide. BZUA is strongly benzoate inducible and subject to CreA-mediated carbon catabolite repression and a probable inactivation of benzoate induction by glucose [32]. A cytochrome P450rm was also isolated from the basidiomycete yeast $R$. minuta as a bifunctional enzyme with isobutene-forming and benzoate 4-hydroxylase activities. The gene product shared $48 \%$ amino acid sequence identity with CYP53A1 from $A$. niger, indicating that the gene belongs to a novel subfamily of CYP53, CYP53B [30].

Here, we used microarray as a means to isolate these two genes, whose expression is upregulated during plant infection, and to identify their transcriptional profile in C. heterostrophus WT and signal deficiency strains. The results may define the unique contribution of each signaling unit to the regulation of distinct biosynthesis pathways. Moreover, the transcriptional profile may point to distinct and shared roles of the signaling components.

\section{Materials and Methods}

\subsection{Strains}

The strains used are listed in Table 1.

\subsection{Sample Preparation for Microarray}

Wild type and signaling mutants were grown in different liquid media: complete medium with xylose (CMX, 48 h) or maltose (CMM, 48 h), minimal medium with xylose (MMX, 72 h) and also inoculated on maize cv. Grand Jubilee (from Pop Vriend Seeds B.V., Andijk, The Netherlands, supplied by Eden Seeds, Reut, Israel) plants (described previously by [11]). The leaves of the plants were dipped into homogenized mycelial suspension in order to obtain widespread lesions. Infected plants were incubated for different periods of time according to virulence of mutant strains. Plants infected with WT or G-protein $\alpha 2$ subunit gene disrupted mutant (cga2) were incubated for 24 hours; during this time, the plant tissue was completely destroyed. Plants infected by chk1, $c g b 1$ and $c g a 1$ were incubated for 48 hours. cga 1 and $c g b 1$ mutants caused a similar degree of infection: a large portion of maize leaf area became necrotic. The chk1 mutant caused the least severe symptoms: leaves acquired yellow coloring and small necrotic lesions appeared. The portion of fungal RNA in samples isolated from infected tissue varies from about 23\% (inoculation by chk1) to 50\% (inoculation by wild type). Ethidium bromide staining of gel-electrophoresed RNA enables relative estimation of plant RNA portion in each sample: the intensity of bands representing plant chloroplast rRNA varies significantly between samples. 
Table 1. Strains used.

\begin{tabular}{|c|c|c|c|}
\hline Strain & Genotype & Reference or Source & Comments \\
\hline WT C4 & MAT1-2; Tox $1^{+}$ATCC 48331 & Leach et al. (1982) & Wild type strains obtained after six backcrosses \\
\hline WT C5 & MAT1-1; Tox1 ${ }^{-}$ATCC 48332 & Leach et al. (1982) & and are nearly isogenic. \\
\hline $\operatorname{cga} 1$ & MAT1-1; Tox1 ${ }^{-}$ATCC 48332 & Horwitz et al. (1999) & $\begin{array}{l}\text { G-protein } \alpha 1 \text { subunit disrupted mutant created by } \\
\text { insertion of the hygromycin cassette into the } \\
\text { coding region combined with an 18-bp deletion. }\end{array}$ \\
\hline $\operatorname{cga} 2$ & MAT1-2; Tox $1^{+}$ATCC 48331 & $\begin{array}{l}\text { Giloh M.Sc. thesis } \\
\text { (cited in Ganem et al., 2004) }\end{array}$ & $\begin{array}{l}\text { G-protein } \alpha 2 \text { subunit disrupted mutant having no } \\
\text { known phenotypes. }\end{array}$ \\
\hline $\operatorname{cgb} 1$ & MAT1-2; Tox $1^{+}$ATCC 48331 & Ganem et al. (2004) & $\begin{array}{l}\text { G-protein } \beta \text { subunit disrupted mutant created by } \\
\text { insertion of the hygromycin cassette into the coding } \\
\text { region combined with a } 473 \text { bp deletion. This strain } \\
\text { contains additional mutations but its phenotypic traits } \\
\text { are similar in almost every aspect to the } c g b 1 \text { that } \\
\text { contains only one mutation. }\end{array}$ \\
\hline chk1 & MAT1-2; Tox $1^{+}$ATCC 48331 & Lev et al. (1999) & $\begin{array}{l}\text { MAPK disrupted mutant created by replacing the } \\
\text { coding region with the hygromycin resistance cassette. }\end{array}$ \\
\hline
\end{tabular}

\subsection{Normalization of Fungal RNA Quantities}

A GAPDH gene (encoding glyceraldehyde-3-phosphate dehydrogenase) fragment generated by PCR with primers 5' CCCTCGCCTGACGCCCCCAT 3' and 5' CGAGGACACGGCGGGAGTAA 3' was used to quantify fungal RNA. Total RNA of mutants and WT (with plant RNA added) infected leaves were the starting material for mRNA isolation using the polyATtract kit (Promega).

\subsection{Microarray Search and Results Processing}

The two selected genes, $B P H$ and $C I P A$, were chosen from the array database (Syngenta Biotechnology Inc., NC USA, Affymetrix Chip containing 25,215 sequences based on 13,309,801 bases) according to their unique expression profile in the various signaling mutants. The array search parameters aimed at finding fungal genes that are upregulated in plant infection in comparison to liquid media growth, i.e., genes with low expression levels in all strains and all media, except for the corn leaves infection experiment. We also defined the search parameter to isolate genes that differ in their expression in one or more mutant strains in comparison to the WT in at least two-fold normalized expression intensity. The selected genes were identified by homology search against the $C$. heterostrophus genome sequence database (genome sequences of $C$. heterostrophus strains C4 and C5 are now available publicly from the JGI website at http://www.jgi.doe.gov/) using TBLASTN [33] and by BLASTX against the gene bank database using the NCBI (National Center for Biotechnology Information, MD, USA) BLAST sequence analysis services at http://www.ncbi.nlm.nih.gov/BLAST/.

\subsection{Phylogenetic Relationships}

Protein sequences were aligned using ClustalX 1.83 (http://www.clustal.org) [34]. Alignment was done using bootstrap N-J tree (1000) and exclude positions with gaps. The TreeView program

(http://taxonomy.zoology.gla.ac.uk/rod/treeview.html) was used to generate a phylogenetic tree; default options were chosen.

\section{Results}

We used two new isolated genes BPH and CIPA to study the role of the G-protein $\alpha$ and $\beta$ subunits and of MAPK (Chk1) in regulating distinct biosynthetic pathways and in controlling target gene expression. These two genes were chosen from the array database (Affymetrix Chip) according to their unique expression profile in the various signaling mutants. The $B P H$ and CIPA genes are upregulated in plant infection in comparison to liquid media growth and are differ in their expression in one or more mutant strains in comparison to the WT in at least two-fold normalized expression intensity.

\subsection{CIPA Regulation by $C$. heterostrophus Signal Transduction G-Protein and MAPK Pathways}

Comparison of the deduced amino acid sequence of the C. heterostrophus CipA protein with the sequences 
present in the databank (BLASTX, NCBI BLAST sequence analysis services) indicated a significant similarity to IfR family protein of A. fumigatus (BLASTX: Expect $=1 \mathrm{e}-54$, in 549 bp). C. heterostrophus CipA share high homology with other IfR proteins from bacteria, fungi and plants (Figure 1). Phylogenetic analysis of 61 known IFR genes products (including the $C$. heterostrophus CipA) revealed the well-conserved distribution of the IfRs into three branches according to the kingdoms with apparently common ancestor (Figures 1 and 2). C. heterostrophus CIPA is located in the phylogenic tree in the fungi branch and is most closely related to the $A$. fumigatus IFR gene (in 869 out of 1000 predicted trees).

Apparently the ancestor carrying the IFR gene for the first time was a bacterium, and this gene was then spread to other bacteria, fungi and plants species in a nonlinear way. Although variations exist in the roots of the phylogenic tree, as expected (for example, 211 out of 1000 predictions in one root that split into bacteria and plant branches, or 149 out of 1000 predictions in the root of the C. heterostrophus IFR gene branch), an obvious similarity exists within the gene families (usually over 600 of the 1000 predictions) (Figure 1). A phylogenic analysis based on the NCBI BLASTX high homology sequences revealed a much more conserved tree (Figure 2). To date, only several IFRL genes have been identified in fungi members, including A. fumigatus, E. nidulans, Candida sp. and N. crassa. Nevertheless, hypothetical proteins with high homology have been identified in other fungi as well.

The CIPA expression profile was examined by microarray analysis (Figure 3). The microarray is a sensitive tool enabling us to compare the amount of transcript levels between the different media and the different signaling disrupted mutant strains. CIPA showed a basal, relatively low, expression level on all four media examined by the microarray analysis, except for in plant infection (Figure 3). RNA samples taken from infected leaves indicate significant differences (four-fold) in the expression levels of the CIPA gene between C. heterostrophus strains. High expression levels of CIPA were observed in WT C4, cga2 and chk1 strains in comparison to cga1 and $c g b 1$ strains. The similar consequence of disruption $c g a 1$ or $c g b 1$ separately may imply a common role for both signaling pathways.

\subsection{BPH Regulation by $C$. heterostrophus Signal Transduction G-Protein and MAPK Pathways}

Comparison of the deduced amino acid sequence of the $C$. heterostrophus second protein tested here, $B P H$, against the sequences present in the databank (BLASTX, NCBI BLAST sequence analysis services) indicated a significant similarity to the superfamily of cytochrome P450 IfR enzymes. C. heterostrophus BPH is a high homolog (BLASTX: Expect $=9 \mathrm{e}-177$, in $1605 \mathrm{bp}$ ) of an A. niger P450 gene, CYP53, which belongs to the P450LIII family [35] [36]. The gene shares high homology with other fungal BPH genes such as E. nidulans, $N$. crassa, P. chrysosporium, R. minuta and Nectria haematococca BPHs (Figure 4). Moreover, many hypothetical proteins in other fungi also show high homology to this gene product (including G. zeae, U. maydis and M. grisea).

Phylogenetic analysis of 55 known cytochrome $\mathrm{P} 450$ genes products (including the $C$. heterostrophus $\mathrm{BpH}$ ) revealed the distribution of the BpHs into several branches according to the organisms' kingdoms with apparently a common bacterial ancestor (Figure 4). It appears that the cytochrome P450 gene was then spread to other bacteria, fungi, plants and animal species in parallel pathways. C. heterostrophus $\mathrm{BpH}$ is located in the phylogenic tree in the fungi branch and is most closely related to Aspergillus sp. $\mathrm{BpH}$ (in 845 out of 1000 predicted trees). It also has a common root with $N$. crassa, Rhodotorula spp. and $U$. maydis $B P H$ genes (in $100 \%$ of the predicted trees). The fungi branch is rooted together with part of the animal cytochrome P450 branch in 301 out of 1000 predicted trees and this shared root is attached to the plant's cytochrome P450 root (in 118 out of 1000 predicted trees) (Figure 4).

Microarray analysis of $C$. heterostrophus $B P H$ expression profile exhibits significant upregulation of this gene when fungi grow on maize plant leaves (in comparison to modified liquid media) (Figure 5). In comparison to the WT, $B P H$ expression on plant leaves increased in the cga 1 strain and decreased in both the cga 2 and cgb1 strains. Under these conditions, chk1 showed normalized $B P H$ expression intensity levels similar to the WT.

\section{Discussion}

Deciphering the signaling mechanisms that lead to fungal development and pathogenicity has been subject to intensive study over the past decade. Although a considerable amount of data exists on the key player in these conserved pathways, the G-protein-encoding genes and the MAPK, less is known about downstream effectors, 


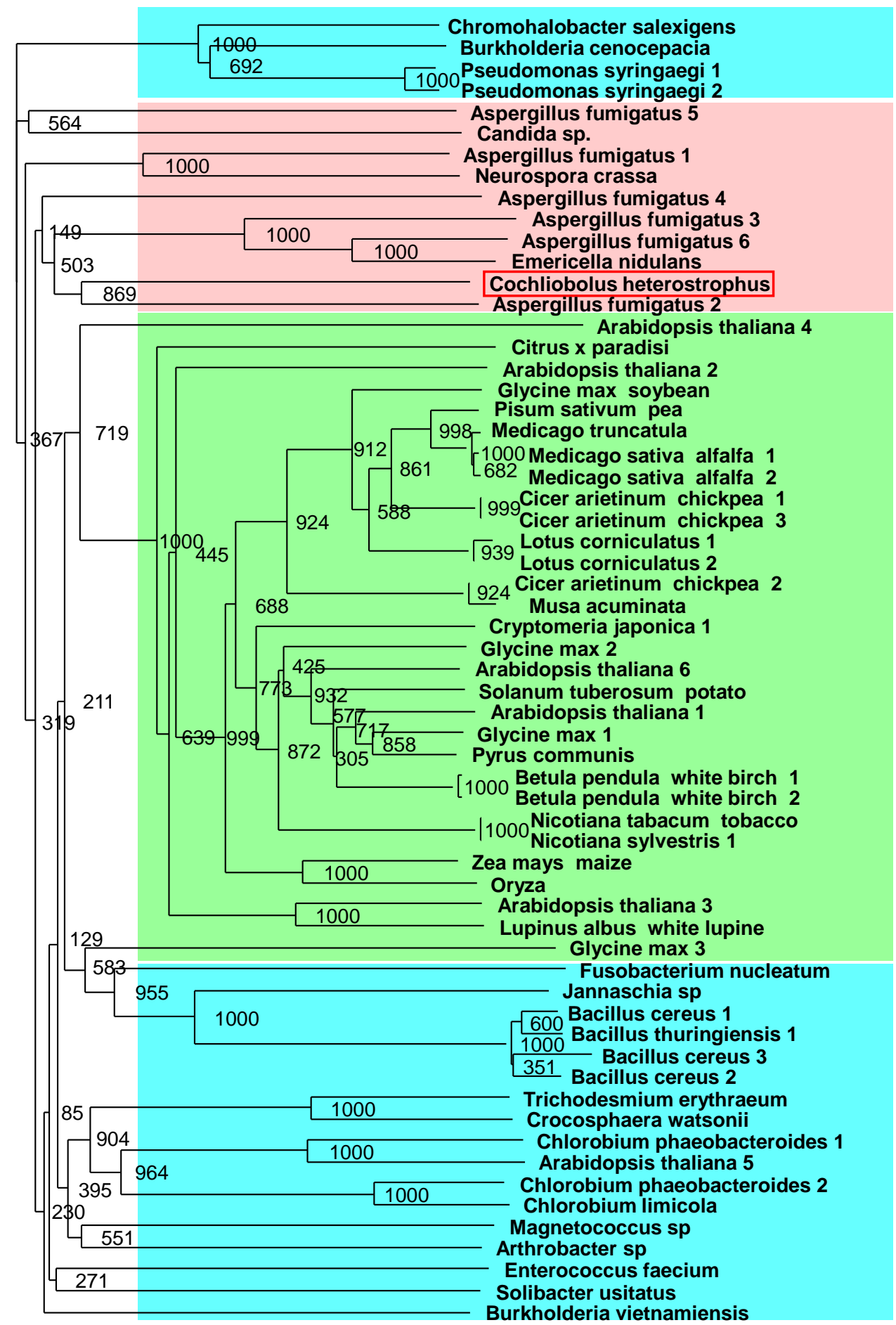

Bacteria

(out-group)

Fungi

Plants

Bacteria

0.1

Figure 1. C. heterostrophus 2'-hydroxyisoflavone reductase (IfR, CipA) phylogenic relationship. The IfR enzyme involved in the synthesis of phytoalexin, which catalyzes an intermediate step in pisatin biosynthesis. Protein sequences were aligned using CLUSTALX 1.83 (Web site: http://www.clustal.org) [34]. Alignment was done using bootstrap N-J tree (1000). The TreeView program (http://taxonomy.zoology.gla.ac.uk/rod/treeview.html) was used to generate a phylogenetic tree; default options were chosen. Square red frame indicates the C. heterostrophus CipA deduced protein. Numbers indicate bootstrap values for each node point. Few bacteria species were defined to be an out group and the root of this tree. 


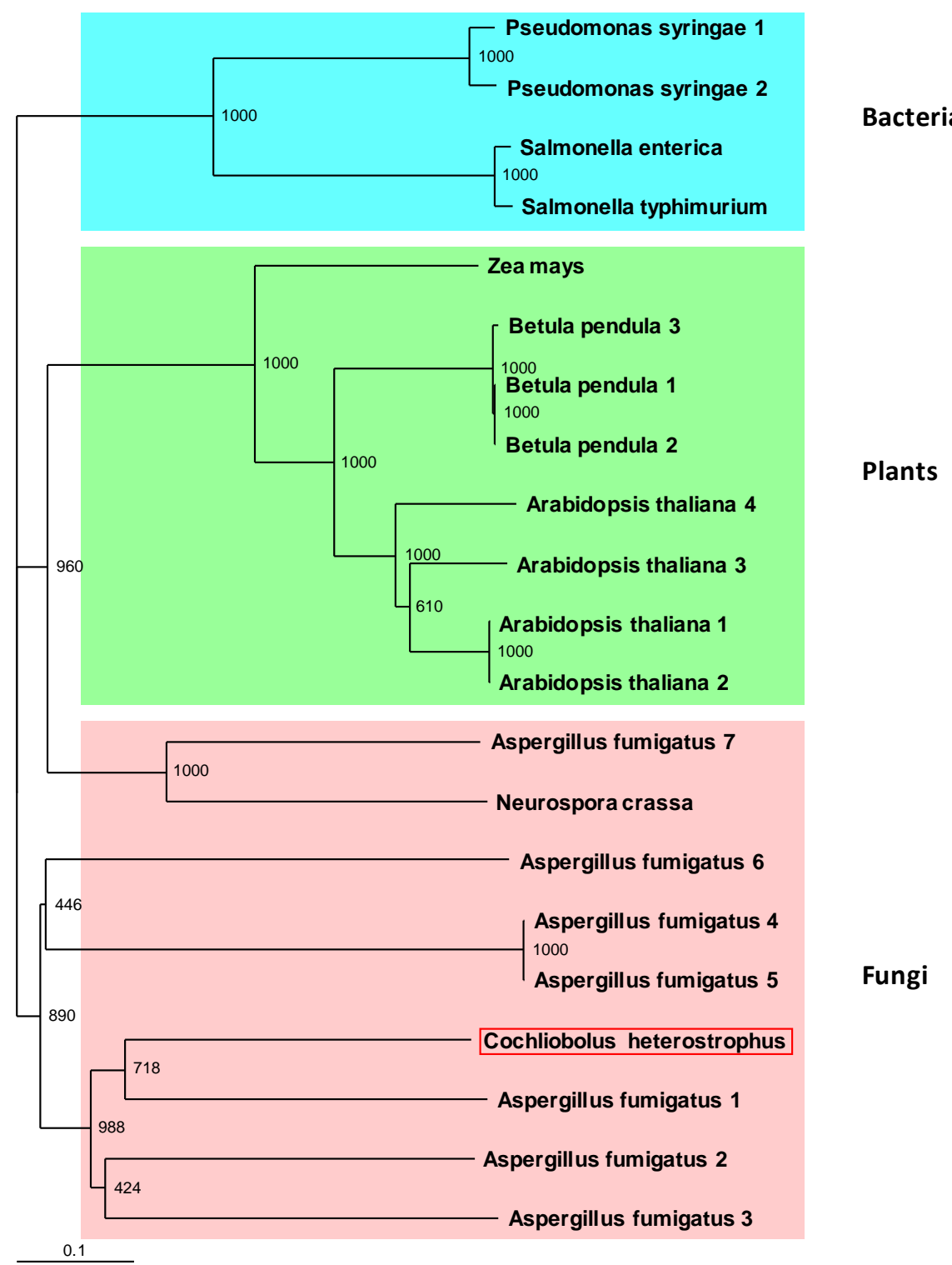

Figure 2. C. heterostrophus CipA phylogenic relationship to closely related sequences. Protein sequences that showed high homology in the BLASTX of C. heterostrophus IfR against the database were aligned using CLUSTALX 1.83, and a phylogenetic tree was generated using the TreeView program as described in Figure 1. Square red frame indicates the $C$. heterostrophus CipA deduced protein. Numbers indicate bootstrap values for each node point.

and the remaining components of these pathways still need to be revealed [37]. At the output of these cascades are sets of target genes, whose study of expression has begun in recent years. We previously identified roles for the individual G-protein $\mathrm{G} \alpha$ and $\mathrm{G} \beta$ subunits [1]-[3] [5] [6] and for MAPK [4] [11] in C. heterostrophus development, reproduction and pathogenicity (summarized in [5]). To date, little knowledge exists on downstream signaling elements of these cascades in this species. The influence on target genes expression was demonstrated in only a few instances [11]-[13].

Here, we identified two genes controlled by the C. heterostrophus signal transduction pathway that are key players in biosynthetic processes. These processes are involved in response to environmental conditions that may include the fungal response to the host as is discussed in detail below. Indeed, the two genes encode for IFRL and $B P H$, and are highly expressed during the infection of the host plant leaves (Figures 3 and 5). 


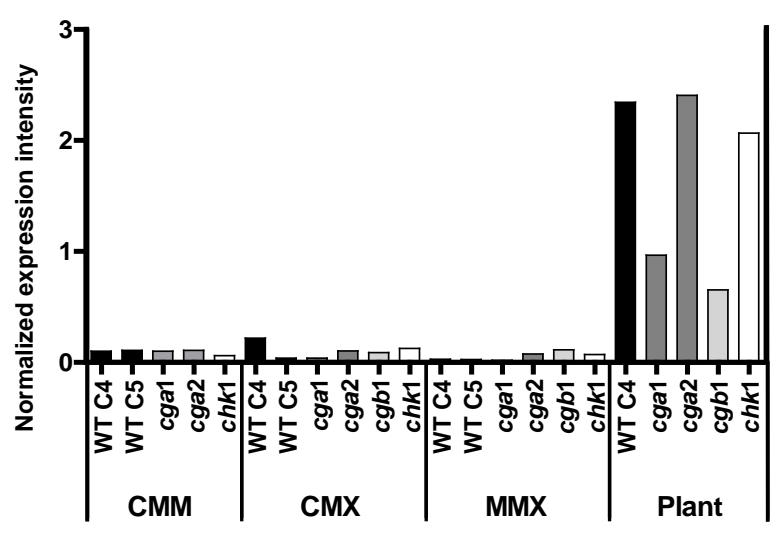

Figure 3. C. heterostrophus CIPA expression profile. Microarray (Affymetrix Chip) normalized expression results indicate transcript levels of WT $(\mathrm{C} 4, \mathrm{C} 5)$ and the signal deficiency mutant strains: cga1 ( $\Delta G \alpha 1)$; cga2 $(\Delta G \alpha 2)$; cgb1 ( $\Delta G \beta 1)$; chk1 ( $\triangle M A P K 1)$. CMM (complete medium with maltose); CMX (complete medium with xylose); MMX (minimal medium with xylose); Plant (plant inoculation).

IfR is an NADPH-dependent reductase involved in the biosynthesis of isoflavonoid phytoalexins in legumes [19]. It is known that plants respond to pathogen attack by activating defense reactions, including changes in ion flux, generation of reactive oxygen species, production of phytoalexins, production of pathogenesis-related proteins and enzymes, and reinforcement of the cell wall [38]. Isoflavonoid phytoalexins are low molecular weight anti-microbial compounds produced by plants in response to pathogen attack, elicitor molecules, or other biotic and abiotic stresses [15]. Obligate mycorrhizal fungi that form mycorrhizae with alfalfa roots apparently suppressed certain host defense reactions. For example, Glomus versiforme suppressed the host defense response in two Medicago spp. by lowering the relative mRNA ratios (standardized to rRNA) of IFR after 40 days [39]. Volpin et al. [40] also suggested that suppression of the normal host isoflavonoid phytoalexin defense response is mediated by changes in RNA transcription. Suppression of the plant defense response by a penetrating microorganism has also been reported in bacterial systems [41]. Bacteria reduces their exposure to isoflavonoids by catabolization of flavonoids [42] and isoflavonoids [43] [44] or by isoflavonoid efflux pumps, as found in rhizosphere bacterium [45].

IfRL proteins have been grouped in a family solely on the basis of their high sequence similarity with IfRs, which catalyze the reduction of $\alpha, \beta$-unsaturated ketones and which are involved in biosynthesis of isoflavonoid phytoalexins in legumes in response to fungal infection [20] [21] [46]. However, IfRL proteins have been identified in plants that do not synthesize isoflavonoid phytoalexins in response to pathogen attack [47], and therefore IfRLs are likely to have broader functions. IfRLs have been implied to function in response to oxidative stress in Arabidopsis [48], prolonged sulfur starvation in maize [49], and UV radiation in harvested grapefruit [50]. It has been suggested that all IfRL proteins are oxidoreductases (EC 1.3.1 that act on the CH-CH Group of Donors) utilizing NAD(P)H as a cofactor, which have various substrates that may or may not be related structurally to flavonoids [48]. Evidence from A. fumigatus/nidulans [23] and Candida sp. [24] indicates that the IFR genes are upregulated in fungi in response to biotic and abiotic stresses. Together with the findings in this work that point to an upregulation of IFRL in response to plant nutritional source (Figure 3), it may be possible that IfR recruits general defense responses for the fungus including host defense induced stresses.

Very little is known about the signal transduction pathways inducing IFRL genes. Kim et al. [51] showed that the induction of OsIRL by the fungal elicitor in plants was blocked by calyculin A, but not affected by staurosporine, suggesting that phosphatase activity is necessary for OsIRL induction, but not hyperphosphorylation. Elicitor-induced OSIRL transcription was also somewhat induced by the calcium ionophore A23187, suggesting that $\mathrm{Ca}^{2+}$ ions are involved. Here, we reported that IFRL expression is controlled in the plant pathogen $C$. heterostrophus by the G-protein signaling pathway, but not the MAPK. In particular, we discovered that G-protein $\alpha$ and $\beta$ subunits are involved in maintaining normal levels of IFRL expression. Disruption of this signaling components results in a significant decrease in IFRL expression (Figure 3). It is likely that this well-known 


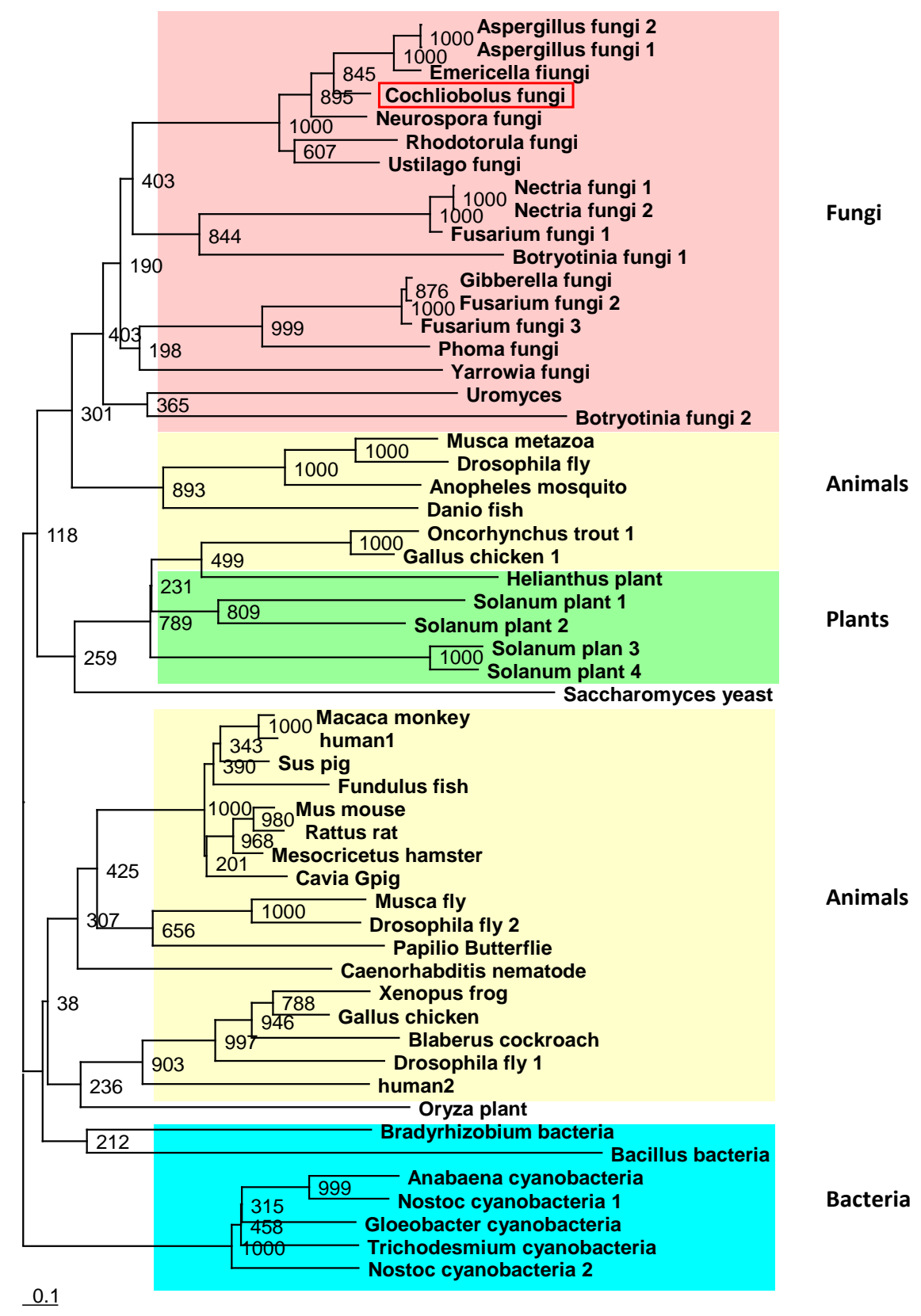

Figure 4. Cytochrome P450 benzoate 4-monooxygenase $(\mathrm{BpH})$ family phylogenic tree. Protein sequences were aligned using CLUSTALX 1.83, and the TreeView program was used to generate a phylogenetic tree, as described in Figure 1. Square red frame indicates the $C$. heterostrophus $B P H$ deduced protein. Numbers indicate bootstrap values for each node point.

environmental sensing mediation mechanism will be involved if IFRL is indeed related to stress response in fungi.

The $B P H$ is another gene that may be a target for signaling mediation control. $B P H$ is a member of cytochrome P450 enzyme systems that are found throughout nature and are involved in many different, often complex, bioconversions [29]. We identified a high homolog to the A. niger BPHA gene in the C. heterostrophus genome. The antimicrobial nature of lipophilic weak acids such as benzoate has been widely used in the preservation of foodstuffs [52]. Benzoate induces an energy-consuming stress response in conjunction with the reduction in glucose fermentation, which leads to a drastic net decrease in cellular ATP levels, reducing the rate of growth of 


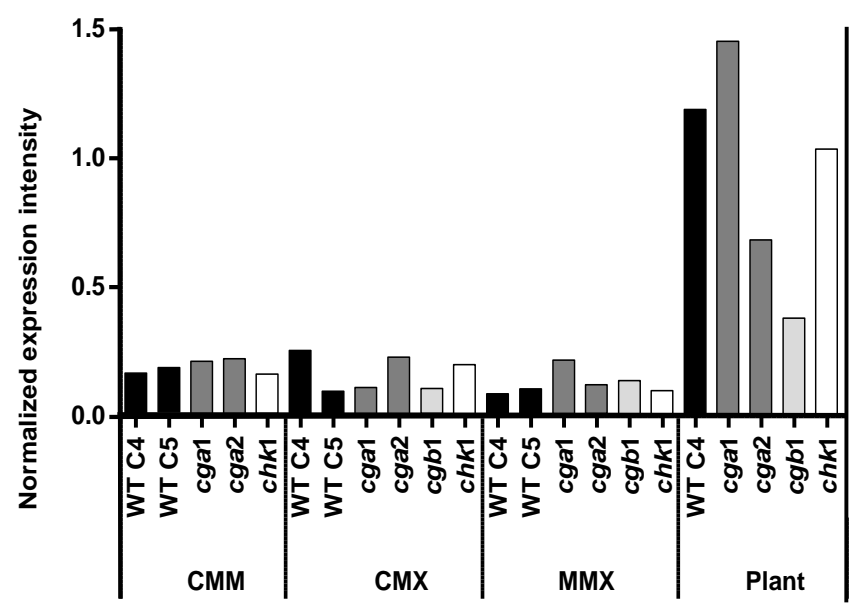

Figure 5. C. heterostrophus BPH expression profile. Microarray (Affymetrix Chip) normalized expression results indicate transcript levels of WT (C4 and C5) and the signal deficiency mutant strains in different media and in maize plants infected leaves. Abbreviations as in Figure 3.

Saccharomyces cerevisiae [53]. Mutation in the BZUA, an A. nidulans ortholog of BPHA from A. niger, led to sensitivity to benzoate, indicating that $\mathrm{BpH}$ plays an essential role in resistance to benzoate stress [32]. In this work, we found an overall induction of $\mathrm{BPH}$ gene expression in $C$. heterostrophus cultures that grew on the host plan tissue in comparison to cultures in modified liquid media (Figure 5). This interesting observation may raise the possibility that the host plant uses some defense mechanism that results in benzoate production. Indeed, infection of tobacco plants with tobacco mosaic virus or elicitation of cells led to a rapid de novo synthesis and accumulation of conjugated benzoic acid [54]. It was concluded that benzoic acid is likely to play a role as precursor of salicylic acid biosynthesis in tobacco plants undergoing a hypersensitive response following infection. Salicylic acid (SA, 2-hydroxybenzoic acid) has been known for some time to be a key endogenous component of local and systemic disease resistance in plants [55]. By using a series of signaling deficiency mutants, we identified possible roles for G-protein subunits in mediating $B P H$ expression in response to the host plant (Figure 5). Microarray analysis indicates induction in $B P H$ transcription levels in cga1, moderate reduction in cga 2 and severe reduction in $c g b 1$. This observation may hint to a common role for $\mathrm{G} \alpha$ and $\mathrm{G} \beta$ in maintaining accurate levels of this enzyme during pathogenesis. Such epistasis relations were observed in the determination of other phenotypic traits [5]. Interestingly, it is the first recognized role for cga2 in this organism, and the opposite function in comparison to cga1 makes this finding even more inquiring. It will be interesting to see if inoculating these strains on benzoate will support these findings.

\section{Conclusion}

The key players in signal transduction pathways, G-protein and MAPK, are mediators of environmental sensing responses that lead, through as yet unknown cascades, to an alteration of target gene expression. This work has shown that these genes, even though they share an environmental dependent response, participate in the regulation of two distinct biosynthesis pathways key players, CipA — a member of the IfR protein family and $B P H-a$ member of cytochrome P450, enzyme systems. Furthermore, the signaling components act as inducers or repressors depending on the target gene and environmental conditions, indicating a complex role. It will be most interesting to understand how the same signal mechanism can fine-tune an enormous number of distinct and sometimes opposite responses and, at the same time, maintain a fine balance with the other signal cascades.

\section{Acknowledgements}

I would like to thank Dr. Benjamin A. Horwitz (Technion-Israel Institute of Technology, Israel) for his guidance and helpful advice, Mary Shahar (Technion-Israel Institute of Technology, Israel) for assisting in the lab procedures and Dr. Sophie Lev for the array database and her many helpful suggestions. 


\section{References}

[1] Degani, O., Maor, R., Hadar, R., Sharon, A. and Horwitz, B.A. (2004) Host Physiology and Pathogenic Variation of Cochliobolus heterostrophus Strains with Mutations in the G Protein Alpha Subunit, CGA1. Applied and Environmental Microbiology, 70, 5005-5009. http://dx.doi.org/10.1128/AEM.70.8.5005-5009.2004

[2] Ganem, S., Lu, S.W., Lee, B.N., Chou, D.Y., Hadar, R., Turgeon, B.G. and Horwitz, B.A. (2004) G-protein Beta Subunit of Cochliobolus heterostrophus Involved in Virulence, Asexual and Sexual Reproductive Ability, and Morphogenesis. Eukaryot Cell, 3, 1653-1663. http://dx.doi.org/10.1128/EC.3.6.1653-1663.2004

[3] Horwitz, B.A., Sharon, A., Lu, S.W., Ritter, V., Sandrock, T.M., Yoder, O.C. and Turgeon, B.G. (1999) A G Protein Alpha Subunit from Cochliobolus heterostrophus Involved in Mating and Appressorium Formation. Fungal Genetics and Biology, 26, 19-32. http://dx.doi.org/10.1006/fgbi.1998.1094

[4] Lev, S., Sharon, A., Hadar, R., Ma, H. and Horwitz, B.A. (1999) A Mitogen-Activated Protein Kinase of the Corn Leaf Pathogen Cochliobolus heterostrophus Is Involved in Conidiation, Appressorium Formation, and Pathogenicity: Diverse Roles for Mitogen-Activated Protein Kinase Homologs in Foliar Pathogens. Proceedings of the National Academy of Sciences of the United States of America, 96, 13542-13547. http://dx.doi.org/10.1073/pnas.96.23.13542

[5] Degani, O. (2013) Cochliobolus heterostrophus G-Protein Alpha and Beta Subunit Double Mutant Reveals Shared and Distinct Roles in Development and Virulence. Physiological and Molecular Plant Pathology, 82, 35-45. http://dx.doi.org/10.1016/j.pmpp.2013.01.004

[6] Degani, O. (2013) Construction of a Constitutively Activated Ga Mutant in the Maize Pathogen Cochliobolus heterostrophus. American Journal of Plant Sciences, 4, 2394-2399. http://dx.doi.org/10.4236/ajps.2013.412296

[7] Clapham, D. and Neer, E.J. (1993) New Roles for G Protein Betagamma Dimers in Transmembrane Signalling. Nature, 365, 403-406. http://dx.doi.org/10.1038/365403a0

[8] Crespo, P., Xu, N., Simonds, W.F. and Gutkind, J.S. (1994) Ras-Dependent Activation of MAP Kinase Pathway Mediated by G-Protein Beta Gamma Subunits. Nature, 369, 418-420. http://dx.doi.org/10.1038/369418a0

[9] Gudermann, T. (2001) Multiple Pathways of ERK Activation by G Protein-Coupled Receptors. Novartis Foundation Symposia, 239, 68-84. http://dx.doi.org/10.1002/0470846674.ch7

[10] Degani, O. (2014) G Protein and MAPK Signaling Pathways Control the Ability of Cochliobolus heterostrophus to Exploit Different Carbon Sources. Advances in Biological Chemistry, 4, 40-50. http://dx.doi.org/10.4236/abc.2014.41007

[11] Lev, S. and Horwitz, B.A. (2003) A Mitogen-Activated Protein Kinase Pathway Modulates the Expression of Two Cellulase Genes in Cochliobolus heterostrophus during Plant Infection. Plant Cell, 15, 835-844. http://dx.doi.org/10.1105/tpc.010546

[12] Degani, O., Lev, S. and Ronen, M. (2013) Hydrophobin Gene Expression in the Maize Pathogen Cochliobolus heterostrophus. Physiological and Molecular Plant Pathology, 83, 25-34. http://dx.doi.org/10.1016/j.pmpp.2013.03.003

[13] Eliahu, N., Igbaria, A., Rose, M.S., Horwitz, B.A. and Lev, S. (2007) Melanin Biosynthesis in the Maize Pathogen Cochliobolus heterostrophus Depends on Two Mitogen-Activated Protein Kinases, Chk1 and Mps1, and the Transcription Factor Cmr1. Eukaryotic Cell, 6, 421-429. http://dx.doi.org/10.1128/EC.00264-06

[14] Dixon, R.A., Dey, P.M. and Lamb, C.J. (1983) Phytoalexins: Enzymology and Molecular Biology. Advances in Enzymology and Related Areas of Molecular Biology, 55, 1-136.

[15] Dixon, R.A. (2001) Natural Products and Plant Disease Resistance. Nature, 411, 843-847. http://dx.doi.org/10.1038/35081178

[16] Wu, Q. and VanEtten, H.D. (2004) Introduction of Plant and Fungal Genes into Pea (Pisum sativum L.) Hairy Roots Reduces Their Ability to Produce Pisatin and Affects Their Response to a Fungal Pathogen. Molecular Plant-Microbe Interactions, 17, 798-804. http://dx.doi.org/10.1094/MPMI.2004.17.7.798

[17] Agrios, G.N. (2005) Plant Pathology Academic Press Inc., London.

[18] Dewick, P.M. (1988) Isoflavonoids. The Flavonoids. Advances in Research since 1980, Chapman and Hall, New York. http://dx.doi.org/10.1007/978-1-4899-2913-6_5

[19] Oommen, A., Dixon, R.A. and Paiva, N.L. (1994) The Elicitor-Inducible Alfalfa Isoflavone Reductase Promoter Confers Different Patterns of Developmental Expression in Homologous and Heterologous Transgenic Plants. Plant Cell, 6, 1789-1803.

[20] Paiva, N.L., Edwards, R., Sun, Y.J., Hrazdina, G. and Dixon, R.A. (1991) Stress Responses in Alfalfa (Medicago sativa L.) 11. Molecular Cloning and Expression of Alfalfa Isoflavone Reductase, a Key Enzyme of Isoflavonoid Phytoalexin Biosynthesis. Plant Molecular Biology, 17, 653-667. http://dx.doi.org/10.1007/BF00037051

[21] Tiemann, K., Inze, D., Van Montagu, M. and Barz, W. (1991) Pterocarpan Phytoalexin Biosynthesis in Elicitor-Chal- 
lenged Chickpea (Cicer arietinum L.) Cell Cultures. Purification, Characterization and cDNA Cloning of NADPH: Isoflavone Oxidoreductase. European Journal of Biochemistry, 200, 751-757. http://dx.doi.org/10.1111/j.1432-1033.1991.tb16241.x

[22] Paiva, N.L., Oommen, A., Harrison, M.J. and Dixon, R.A. (1994) Regulation of Isoflavonoid Metabolism in Alfalfa. Plant Cell, Tissue and Organ Culture, 38, 213-220. http://dx.doi.org/10.1007/BF00033879

[23] Melin, P., Schnurer, J. and Wagner, E.G. (2002) Proteome Analysis of Aspergillus nidulans Reveals Proteins Associated with the Response to the Antibiotic Concanamycin A, Produced by Streptomyces Species. Molecular Genetics and Genomics, 267, 695-702. http://dx.doi.org/10.1007/s00438-002-0695-0

[24] Hong, Y.M., Park, S.W. and Choi, S.Y. (1998) Expression of the CIP1 Gene Induced under Cadmium Stress in Candida sp. Molecules and Cells, 8, 84-89.

[25] Nelson, D.R., Koymans, L., Kamataki, T., Stegeman, J.J., Feyereisen, R., Waxman, D.J., Waterman, M.R., Gotoh, O., Coon, M.J., Estabrook, R.W., Gunsalus, I.C. and Nebert, D.W. (1996) P450 Superfamily: Update on New Sequences, Gene Mapping, Accession Numbers and Nomenclature. Pharmacogenetics, 6, 1-42. http://dx.doi.org/10.1097/00008571-199602000-00002

[26] Omura, T. (1999) Forty Years of Cytochrome P450. Biochemical and Biophysical Research Communications, 266, 690-698. http://dx.doi.org/10.1006/bbrc.1999.1887

[27] Nelson, D.R. (1999) Cytochrome P450 and the Individuality of Species. Archives of Biochemistry and Biophysics, 369, 1-10. http://dx.doi.org/10.1006/abbi.1999.1352

[28] Gonzalez, F.J. (1990) Molecular Genetics of the P-450 Superfamily. Pharmacology and Therapeutics, 45, 1-38. http://dx.doi.org/10.1016/0163-7258(90)90006-N

[29] Graham, S.E. and Peterson, J.A. (1999) How Similar Are P450s and What Can Their Differences Teach Us? Archives of Biochemistry and Biophysics, 369, 24-29. http://dx.doi.org/10.1006/abbi.1999.1350

[30] Fujii, T., Nakamura, K., Shibuya, K., Tanase, S., Gotoh, O., Ogawa, T. and Fukuda, H. (1997) Structural Characterization of the Gene and Corresponding cDNA for the Cytochrome P450rm from Rhodotorula minuta Which Catalyzes Formation of Isobutene and 4-Hydroxylation of Benzoate. Molecular and General Genetics, 256, 115-120. http://dx.doi.org/10.1007/s004380050552

[31] Van den Brink, J.M., Punt, P.J., van Gorcom, R.F. and van den Hondel, C.A. (2000) Regulation of Expression of the Aspergillus niger Benzoate Para-Hydroxylase Cytochrome P450 System. Molecular and General Genetics, 263, 601609. http://dx.doi.org/10.1007/s004380051207

[32] Fraser, J.A., Davis, M.A. and Hynes, M.J. (2002) The Genes gmdA, Encoding an Amidase, and bzuA, Encoding a Cytochrome P450, Are Required for Benzamide Utilization in Aspergillus nidulans. Fungal Genetics and Biology, 35, 135-146. http://dx.doi.org/10.1006/fgbi.2001.1307

[33] Altschul, S.F., Gish, W., Miller, W., Myers, E.W. and Lipman, D.J. (1990) Basic Local Alignment Search Tool. Journal of Molecular Biology, 215, 403-410.

[34] Thompson, J.D., Gibson, T.J., Plewniak, F., Jeanmougin, F. and Higgins, D.G. (1997) The CLUSTAL_X Windows Interface: Flexible Strategies for Multiple Sequence Alignment Aided by Quality Analysis Tools. Nucleic Acids Research, 25, 4876-4882. http://dx.doi.org/10.1093/nar/25.24.4876

[35] Boschloo, J.G., Moonen, E., van Gorcom, R.F., Hermes, H.F. and Bos, C.J. (1991) Genetic Analysis of Aspergillus niger Mutants Defective in Benzoate-4-Hydroxylase Function. Current Genetics, 19, 261-264. http://dx.doi.org/10.1007/BF00355052

[36] Van Gorcom, R.F., Boschloo, J.G., Kuijvenhoven, A., Lange, J., van Vark, A.J., Bos, C.J., van Balken, J.A., Pouwels, P.H. and van den Hondel, C.A. (1990) Isolation and Molecular Characterisation of the Benzoate-Para-Hydroxylase Gene (bphA) of Aspergillus niger: A Member of a New Gene Family of the Cytochrome P450 Superfamily. Molecular and General Genetics, 223, 192-197.

[37] Bolker, M. (1998) Sex and Crime: Heterotrimeric G Proteins in Fungal Mating and Pathogenesis. Fungal Genetics and Biology, 25, 143-156. http://dx.doi.org/10.1006/fgbi.1998.1102

[38] Staskawicz, B.J., Ausubel, F.M., Baker, B.J., Ellis, J.G. and Jones, J.D. (1995) Molecular Genetics of Plant Disease Resistance. Science, 268, 661-667. http://dx.doi.org/10.1126/science.7732374

[39] Harrison, M.J. and Dixon, R.A. (1993) Isoflavonoid Accumulation and Expression of Defense Gene Transcripts during the Establishment of Vesicular-Arbuscular Mycorrhizal Associations in Roots of Medicago truncatula. Molecular Plant-Microbe Interactions, 6, 643-654. http://dx.doi.org/10.1094/MPMI-6-643

[40] Volpin, H., Phillips, D.A., Okon, Y. and Kapulnik, Y. (1995) Suppression of an Isoflavonoid Phytoalexin Defense Response in Mycorrhizal Alfalfa Roots. Plant Physiology, 108, 1449-1454.

[41] Jakobek, J.L., Smith, J.A. and Lindgren, P.B. (1993) Suppression of Bean Defense Responses by Pseudomonas syrin- 
gae. Plant Cell, 5, 57-63.

[42] Rao, J.R. and Cooper, J.E. (1994) Rhizobia Catabolize Nod Gene-Inducing Flavonoids via C-Ring Fission Mechanisms. Journal of Bacteriology, 176, 5409-5413.

[43] Funnell, D.L., Matthews, P.S. and Van Etten, H.D. (2002) Identification of New Pisatin Demethylase Genes (PDA5 and PDA7) in Nectria haematococca and Non-Mendelian Segregation of Pisatin Demethylating Ability and Virulence on Pea Due to Loss of Chromosomal Elements. Fungal Genetics and Biology, 37, 121-133. http://dx.doi.org/10.1016/S1087-1845(02)00503-0

[44] Matthews, D.E. and Van Etten, H.D. (1983) Detoxification of the Phytoalexin Pisatin by a Fungal Cytochrome P-450. Archives of Biochemistry and Biophysics, 224, 494-505. http://dx.doi.org/10.1016/0003-9861(83)90237-0

[45] Palumbo, J.D., Kado, C.I. and Phillips, D.A. (1998) An Isoflavonoid-Inducible Efflux Pump in Agrobacterium tumefaciens Is Involved in Competitive Colonization of Roots. Journal of Bacteriology, 180, 3107-3113.

[46] Dixon, R.A., Harrison, M.J. and Paiva, N.L. (1995) The Isoflavonoid Phytoalexin Pathway: From Enzymes to Genes to Transcription Factors. Physiologia Plantarum, 93, 385-392. http://dx.doi.org/10.1111/j.1399-3054.1995.tb02243.x

[47] Dixon, R.A., Dey, P.M. and Lamb, C.J. (1983) Advances in Enzymology and Related Areas in Molecular Biology. John Wiley \& Sons, New York.

[48] Babiychuk, E., Kushnir, S., Belles-Boix, E., Van Montagu, M. and Inzé, D. (1995) Arabidopsis thaliana NADPH Oxidoreductase Homologs Confer Tolerance of Yeasts toward the Thiol-Oxidizing Drug Diamide. Journal of Biological Chemistry, 270, 26224-26231. http://dx.doi.org/10.1074/jbc.270.44.26224

[49] Petrucco, S., Bolchi, A., Foroni, C., Percudani, R., Rossi, G.L. and Ottonello, S. (1996) A Maize Gene Encoding an NADPH Binding Enzyme Highly Homologous to Isoflavone Reductases Is Activated in Response to Sulfur Starvation. Plant Cell, 8, 69-80.

[50] Lers, A., Burd, S., Lomaniec, E., Droby, S. and Chalutz, E. (1998) The Expression of a Grapefruit Gene Encoding an Isoflavone Reductase-Like Protein Is Induced in Response to UV Irradiation. Plant Molecular Biology, 36, 847. http://dx.doi.org/10.1023/A:1005996515602

[51] Kim, S.T., Cho, K.S., Kim, S.G., Kang, S.Y. and Kang, K.Y. (2003) A Rice Isoflavone Reductase-Like Gene, OsIRL, Is Induced by Rice Blast Fungal Elicitor. Molecules and Cells, 16, 224-231.

[52] Booth, I.R., Kroll, R.G. and Gould, G.W. (1989) The Preservation of Foods by Low pH. Mechanisms of Action of Food Preservation Procedures, Elsevier Applied Science, Elsevier Science Publishers Ltd. London, 119-160.

[53] Pearce, A.K., Booth, I.R. and Brown, A.J. (2001) Genetic Manipulation of 6-Phosphofructo-1-Kinase and Fructose 2,6-Bisphosphate Levels Affects the Extent to Which Benzoic Acid Inhibits the Growth of Saccharomyces cerevisiae. Microbiology, 147, 403-410.

[54] Chong, J., Pierrel, M.A., Atanassova, R., Werck-Reichhart, D., Fritig, B. and Saindrenan, P. (2001) Free and Conjugated Benzoic Acid in Tobacco Plants and Cell Cultures. Induced Accumulation upon Elicitation of Defense Responses and Role as Salicylic Acid Precursors. Plant Physiology, 125, 318-328. http://dx.doi.org/10.1104/pp.125.1.318

[55] Shah, Y. and Klessig, D.F. (1999) Salicylic Acid: Signal Perception and Transduction. Elsevier Science, Amsterdam. 